TUMOR OF THE PELVIS

\section{IN A CHILD.}

TO THE EDITORS OF THE RROVINCIAL MEDICAL JOURNAL.

Gentlemen,-If you think the following case possesses sufficient interest to find a place in your Journal, you will much oblige by its insertion,

Your obedient Servant,

Chiarles Hodgkins, M.R.C.S, \&c.

The parents of W. B., aged seven years, about the middle of November last, observed that, after playing, he came into the house walking in a bent position and complaining of having hurt his belly; but, as no extemal bruise appeared, they did not think proper to apply for medical aid, and he seemed soon to regain his cheerfulness. He, however, complained in a few days of uneasiness, and of difficulty in passing his urine, getting up frequently in the night and straining very much before he could succeed; this continued for a fortnight, when, the symptoms becoming urgent, I was sent for on the 1st of December.

I found him lying on a sofa, the body bent forwards and the legs drawn up, complaining of great pain in the belly, great heat, thirst, and other febrile symptoms, with constipation of a fortnight's duration, and passing very little water. Thinking, probably, the loaded state of the bowels was the source of all the complaint, I merely prescribed a brisk purgative, with fomentations to the belly; the following day the bowels had acted, but the urine had decreased in quantity. I now ordered another cathartic and anodyne fomentations.

Dec. 3. A vast quantity of fluid stools mixed with scybalæ had passed; the febrile symptoms less, but the bladder had ceased to act; I therefore introduced the catheter, and drew off three pints of strong smelling urine.

On the 5th, the retention still continuing, I proceeded to examine the case more minutely. On introducing the finger into the rectum, the coats of the bladder appeared to be very much thickened, but I failed to detect any tumor; the abdomen appeared full and rather tender; no particular viscus seemed to be the seat of disease.

8. During the last two days the child objected to the introduction of the catheter, as the urine had dribbled away frequently and involuntarily; but the distension now being painful, he wished it drawn off, after which it was done daily. I now supposed that the bladder might be paralysed from orer distension, and endeavoured to introduce a gum catheter, intending to leave it in, but failed, and the patient decidedly objected to the metallic one being left in.

14. To day I again examined per rectum, and found what appeared to be a large tumor occupying the situation of the prostate gland; the general fulness of the abdomen had increased; the bowels continued to act regularly. I now prescribed remedies to reduce the tumor-such as the hydriodate of potass; \&c. (leeches were objected to); nevertheless, the disease continued to increase, and by January loth the fundus vesica appeared on a level with the umbilicus; the testes were retracted; he began to emaciate; and the appetite became capricious.

January 25. Disease still progresses; he is much emaciated; the belly very large; one leg (the left) œdematous; the water drawn off has acquired a strong fæcal odor; the bladder appears above the umbilicus nearly as high as the pit of the stomach, and a hard substance can be felt across the hypogastrium bchind the bladder; this increased so rapidly that a medical friend who visited him with me supposed there was extensive hepatic enlargement. All the symptoms continued to increase till the 2nd of February, when he died.

Post-mortem Examination Twenty-four Hours after Death.

On laying open the abdomen, the bladder appeared occupying a very prominent situation, the neck being seen above the pubis, the fundus in front of the arch of the colon and as high as the inferior curvature of the stomach; its coats were much thickened and very varicose; there was a black patch, as if gangrenous, the size of a half-crown, on the posterior surfacc; it contained about two pints of urine. The peritoneal cavity contained about a pint of serum of a very fæcal odor; behind the bladder a large tumor appeared, occupying the whole cavity of the pelvis; it was firm to the touch, and so completely did it fill up the pelvis that I had great difficulty in inserting a finger between it and the bones, and naturally created surprise that the patient should have been able to pass the fæces. On raising it out of the pelvis $I$ found that it was attached to the bladder in front and to the rectum behind; it joined the bladder about an inch above the entrance of the ureters, occupying the whole space of the trigonum to the apex; the shape was precisely that of the prostate gland; the length was seven inches and the breadth four and a half or five inches; it weighed between three and four pounds; on the posterior surface was a depression or groove, along which the rectum was attached, which enabled the fæces to pass. The ureters, which were distended to the size of a finger, entered it about the middle; on cutting into it the tumor appeared like the structure of the prostate seen through a magnifying glassspongy in texture and easily broken down-its color was very much that of fresh Castile soap. All the other viscera were healthy, but pushed upwards, the liver being quite up in the chest, and the stomach behind the sternum. The head was not examined.

Bilston, Feb. 8, 1843.

\section{SCROFULOUS ULCERATION OF FACE.}

TO THE EDITORS OF THE RROVINCIAL MEDICAL JOURNAL.

Gentlemen, - I beg to forward the report of the following case for insertion in the Provincial Medical Journal :-

Martha Fowles, forty-five years of age, has been afflicted with scrofula for twenty-three years ; part of her nose had been eaten away, and the affection was extending over the neck and forehead when I accidentally met her in the street, and undertook at my own expense to cure her. I mention this because she said to me, "I cannot afford any more money; I have been to every one almost, and I am sure there is nothing to be done for me." I promised her an effectual cure in three months, which has been effected 
by the administration of the following medicine, with its accompaniment :-

Hydriodate of potass, two drachms;

Water, one pint. A tablespoonful twice a-day.

The nitric oxide of mercury ointment to be used at the time of taking the medicine.

This mode of trcatment was persevered in, and the woman now (after having left off taking medicine four months), instead of being a disagreeable object to every one as well as herself, presents as clean and clear a face as either you or me.

I am, Gentlemen, Yours, \&c.,

HARRY R. LaRKE.

Whitchurch, Salop, Jan. 21, 1843.

\section{CASE}

of

\section{STRANGULATED FEMORAL HERNIA-} OPERATION.

\section{By J. D. Owens, Esq., M.R.C.S.L.}

The number of persons of both sexes that suffer from hernia, and the danger at all times to be apprehended when the protruded bowel cannot be returned by the means usually employed, render it necessary to give more than ordinary attention to this class of diseases ; and the anxiety and alarm of the patient and friends, aroused by the worst anticipations, the baffled endeavours to afford relief, and the hopelessness that seems in prospect, certainly require the exercise of much firmness and intrepidity, and an exact and confident diagnosis. Yet, happily, although so frequently occurring, from the facility with which the intestine is reduced in most instances, it is rarely required to perform the operation-particularly in provincial practice; therefore, in the present case, when the operation was required, the report may be of some interest. It will be observed that the symptoms were obscure throughout, and the strangulation, that existed seven days, so subdued and hidden as hardly to indicate the impending mischief. However, the function of the bowels being obstructed, the sympathetic pains so often complained of, the nausea and sickness more or less persistent, were themselves prominent symptoms of strangulation, but perhaps the peculiarly tense and resisting nature of the tumor conveyed to the practised hand a sensation almost convincing. The operation revealed a segment of intestine protruded through the saphenal aperture, incarcerated by adhesions of long standing, and the stricture bound it so rigidly that not even the flatus it contained could be expelled; at the same time the circulation continued unimpeded, the pains in the part were only slight and transient, and the healthy condition of the viscera remained unchanged.

Dec. 26. I was early this morning requested to visit Mrs. Martha Hughes, Newton's-green, near Craven Arms. The messenger stated her case as being one of twisted bowels, and that she had been without an evacuation for three days. She had a femoral hernia of some years' standing, and a few months preceding her present attack had sought assistance when there existed incipient symptoms of strangulation. The bowel, however, returned sponta- ncously. The histury she is able to give of her case is unconnecled and imperfect. Has noticed the tumor in the groin often, but it did not trouble her. 'Ihinks it had been always down for some time past; whenever she felt the lock (even when lying down) remembers the kernel was there. Is much nauseated, and vomiting frequently a greenish dark fluid; it is mixed with bile and gastric secretions, but free from stercoraceous odor; she, however, represents the earlier ejections as being very fœtid. The hernia in the left groin, the size of a walnut, protrudes from behind Poupart's ligament, is of globular shape, and so tense and firm that the finger cannot impress it; the position of the patient does not affect it, nor docs it receive any impulse from coughing. No constriction nor dragging experienced in the abdomen, but erratic pains are ranging about the parts. Employed the taxis; the direction of the patient was so inclined as to relax muscular resistance when compressing the tumor between the fingers and gently urging it downwards from over the falsiform margin, with the other hand it was kneaded inwards and upwards in the line of canal. The attempt was unsuccessful.

Seven, p.m. Castor oil given in the morning was rejected unchanged almost immediately, and a persistent nausea induced. From time to time the slight wandering pains were present, but the taxis again applied was borne pleasantly. According to some late writers, the elevation of the extremities and full doses of opium have done wonders; here they failed; the parts were guarded from active inflammation arising, by a refrigerating mixture applied in a bladder.

27. Had a restless and weary night; slept a few early hours, but sickness and transitory pains had caused her much disturbance this morning; bowels unevacuated; pulse small and fast; tongue white; the glance of the hand on the abdomen causes her to shrink away, but the pain is relieved by firmly directed pressure; the taxis again failed; hitherto the mure active and decisive measures had been in abeyance. The patient being sixty-eight years of age, affected with chronic tarsal ophthalmia, and greatly harassed by an old asthma, it seemed judicious to delay as long as quite safe the severer claims on her constitution; but the peculiar condition of the tumor and the eccentric sensitiveness already noticed, rendered further delay hazardous. I therefore bled her from a large orifice to fainting, placed her in a warm bath, injected infusion of tobacco in small quantities, increased until the system was fully influenced, perseveringly using the taxis; and, as these means failed, I announced an operation as inevitable. Eight, p.m.: A consultation was held; the physician considered the case as one of simple incarceration, and gave a favorable prognosis. Prescribed opium and a truss with a hollow pad; could only reiterate the danger and trust to time; the smallness of the hernia augured unfavorably.

28. Restless night; disturbed and anxious mind; the morbid insensibility of the peritoneum recurring at short intervals. Warm flannels placed beneath her linen gave relief; gave a dose of castor oil, and administered a turpentine enema; the latter was dejected, immediately bringing with it the contents of the rectum in a scybalous mass; the oil was rejected in six hours by severe romiting, it caused much pain 